\title{
Blockchain-based Education Project
}

\author{
Edward Guustaaf ${ }^{1}$, Untung Rahardja ${ }^{2}$, Qurotul Aini ${ }^{3}$, Herliana Wahyu Maharani ${ }^{4}$, \\ Nesti Anggraini Santoso ${ }^{5}$ \\ ${ }^{1}$ Information Technology, Jakarta, Vexanium \\ ${ }^{2}$ Technical Information, Tangerang, Universitas Raharja \\ 2,3,4,5 Information Systems, Tangerang, Universitas Raharja \\ Email: edward@vexanium.com ${ }^{1}$, untung@raharja.info², aini@raharja.info ${ }^{3}$, \\ herlianamaharani@raharia.info4,
}

\begin{abstract}
To cite this document :
Guustaaf, E., Rahardja, U., Aini, Q., Maharani, H., \& Santoso, N. (2021). Blockchain-

based Education Project. Aptisi Transactions on Management (ATM), 5(1), 46-61.
\end{abstract}

DOI :

https://doi.org/10.33050/atm.v5i1.1433

\section{Abstract}

Blockchain is a jointly distributed and decentralized ledger, in which nodes are aiming to record transaction history with very different networks. This technology is quite practical if used in the field of higher education to carry out digital certification, record keeping, etc. From several papers published on this, no single paper covering educational projects based on the blockchain can find it. So there are the latest opportunities for higher education trends. Projects in the field of higher education that are blockchain-based are aimed at solving problems that occur in educators today. On that basis, we conclude that there is a need for a systematic literature review. Therefore, based on the education project, this study will review the artistic gap between the two. This paper will focus on the protocol used in this project and explore several blockchain-based higher education projects that will be the aim of this paper. As well as analyzing the blockchain features currently in use today and the services that will be offered by existing educational projects, to improve the implementation of technology in the field of education by using the blockchain feature.

Keywords: Blockchain, Education, Digital Certificate, Technology

\section{Introduction}

Bitcoin Crypto Currency is a blockchain technology that was first developed. Since the idea was created in 2008 it has attracted attention, so it became an attraction in the blockchain [1]. Blockchain technology is a technology that can be set itself and decentralized [2] and make open records that have been executed from computerized events or all transactions and will be shared with participating parties [3]. To show the authenticity of every transaction on the blockchain, it will be verified with a digital signature. Information stored on the blockchain will be sealed and cannot be changed, due to the use of digital signatures and encryption [4].

This innovative technology is used in several applications such as education, business industry, health care, and governance because all transactions implemented in the blockchain are transparent, unchanging, and security [5], [6], [7]. In carrying out this technology, security is needed, so that users do not worry about the privacy of the data they provide [8]. One can see the future of blockchain technology in the current era as a developing technology, due to the increasing interest in information and communication technology globally, this has led to changes in all fields including education [9], [10]. In the interest of technology, blockchain technology is felt to have a positive effect [11].

In this era too, blockchain is burning the issue of education and then providing the right solution for them and highlighting the main issues. The certificate is a conventional method of displaying values on paper that is considered to depict a picture of a person. Printing a digital 
certificate is giving the latest trends from current blockchain technology [12]. The possibility of a paper certificate, there is a digital certificate then can already process management simply [13]. Blockchain technology provides a fair mechanism with a transparent nature, complete to fund educational projects and grants, and can improve evaluation systems, salaries for teachers, improve scholarship calculation systems aimed at students, as well as being a cause of the lack of advanced fraud that occurs [14]. The security of blockchain can also be used in the management process of a scientific journal as a support for the security of journal management itself [15]. Various educational projects are introduced for the benefits that users will get, this is done to change the current education system, but there are things we must know and see that in a single study not discussed primarily about this educational project. The latest educational trends can be illustrated through existing gaps. The latest education trends provide insights into the gap between the block-chain and its implementation. This paper will explain some vital education protocols based on blockchain and explain and discuss some of the existing projects to fill this gap.

It also enhances the blockchain features offered and used by existing education to improve the implementation of blockchain technology in education by using blockchain features. Some important blockchain education models from 2013 to 2018 are complete in the conclusions of this paper. Some of the projects are the sony global education, base grad, edgecoin, origin stamp, and teach-me projects. The problem of scalability is one of several problems [2], [5], [16]. The agreed scalability is defined as the time taken to reach consensus and decide on transactions within a block.

Once divided, there will be 5 sections in this paper. An explanation of the features of the blockchain and literature review will be in section 2. Section 3 will contain an exploration of the research method, some research questions, and how the strategies will be used in the search mentioned. Whereas section 4 will cover the results obtained from the research questions and the discussion of our review will be covered in the last section.

\title{
2. Review of Literature
}

In this part of the paper, we will explain some theories and ideas related to studies in the field of blockchain-based education projects and observe some of the block-chains that are its main features. This can build the correlation that occurs between existing secondary studies.

\section{A. Blockchain}

Data records that have become part of the blockchain and have been hosted on decentralized networks cannot be changed, this is simply what blockchain means [7]. Blockchain is peer to peer, where data that has been distributed will be safe in cryptography [17]. All records are almost impossible to update or even damage records on all network objects because all existing records are handled by a group of nodes that cannot be owned by one entity. All transactions that will be brought by the block are bound to each other so that it can be verified and signed by using cryptographic evidence that has been solved by the blockchain and its volunteers. The initial step in the node is to form a block in which there are many transactions. The block will be inspected by almost half the nodes on the network for verification. The blocks that have been verified will be put into chains that have been previously archived by all systems, the purpose of which is not only to make a unique historical record but to make a record of evidence of damage [18], [19].

\author{
B. Types of blockchain \\ Three basic types of blockchain, namely:
}

\section{Allowed blockchain:}

Not being able to issue transactions that they do themselves see their records and join a free community, is an act of blockchain that is permitted and will act as a closed ecosystem. Blockchain access will require strength in the network section for its inner business, centralized organization that makes this access much needed. Personal blockchain also requires being employed by a 
hiring consortium, which is used to request transactions so that they can run safely and be able to share facts with each other.

\section{Blockchain not permitted:}

In this blockchain, it is public or open to anyone who wants to access it. This results in a connection with the user, linking with the existing transfer, and also a complaint that can be made non-public with other users.

\section{Hybrid Blockchain:}

This blockchain uses a combination of two blockchains, namely public blockchain, and private blockchain. On this network blockchain data that is not permitted can still be accessed from the blockchain by using certain stored permissions. This type of blockchain is not open to everyone, but this blockchain provides stealthy basic features such as traceability, integration, and security

\section{Blockchain feature}

Some basic blockchain features, namely:

Decentralized: Blockchain is a record that is open to the public, in which there are nodes, where all nodes are related to each other in the network [5], [6]. Control on the blockchain does not lie in government power, but is decentralized. This decentralization refers to the procedures for storing, preserving, confirming data and transmitting, which occur in the blockchain depending on the structure of the distribution framework [20].

Traceability: All transaction data available on the blockchain will be grouped by indexing the data into sequential order, and one existing block will be linked to two adjacent blocks by cryptographic hashes. All existing block-chains will be investigated through cloud-based sites, the site will be awarded after the mining pool maintains the total block-chain [5], [21], [22].

Consensus Mechanism: The consensus mechanism is used in the blockchain and pc framework, to reach agreement on a single data in a situation where the network is between multiagent frameworks or distributed processes. This mechanism is very helpful in the recording process. There are several procedures in this mechanism namely DPOS, POW, and POS [5], [23].

Currency: In the current revolutionary era, of all the existing cryptocurrency proposals, bitcoin is the first digital currency in the world. Blockchain technology, which is a virtual or digital currency, where this technology ensures end-to-end transactions that make these transactions protected and can be trusted, this is part of the cryptocurrency property. When forming these currencies different development algorithms are used. Therefore, the combined product of cryptocurrency with blockchain can be used in several aspects, for example financial, and accounting aspects [5], [21].

Smart Contract: It is a blockchain protocol needed to enable developers to be able to code financial agreements on the blockchain, which will later be activated by all parties involved [5], [21], [22]. This contract ensures the quality and security of transactions significantly, not only reducing the external costs that are in conventional transactions. This smart contract was built to secure transactions that occur within the blockchain, in the mid-1990s this protocol was first developed by Nick Zabo [24].

Immutability: if data has been entered, then the data cannot be changed again [25]. The existing data is also impossible to manipulate, because it is controlled by all the people concerned If you want to do damage (make changes to be invalid) then you need to change the records that have been stored in the system by more than 51 percent [5], [21]. 


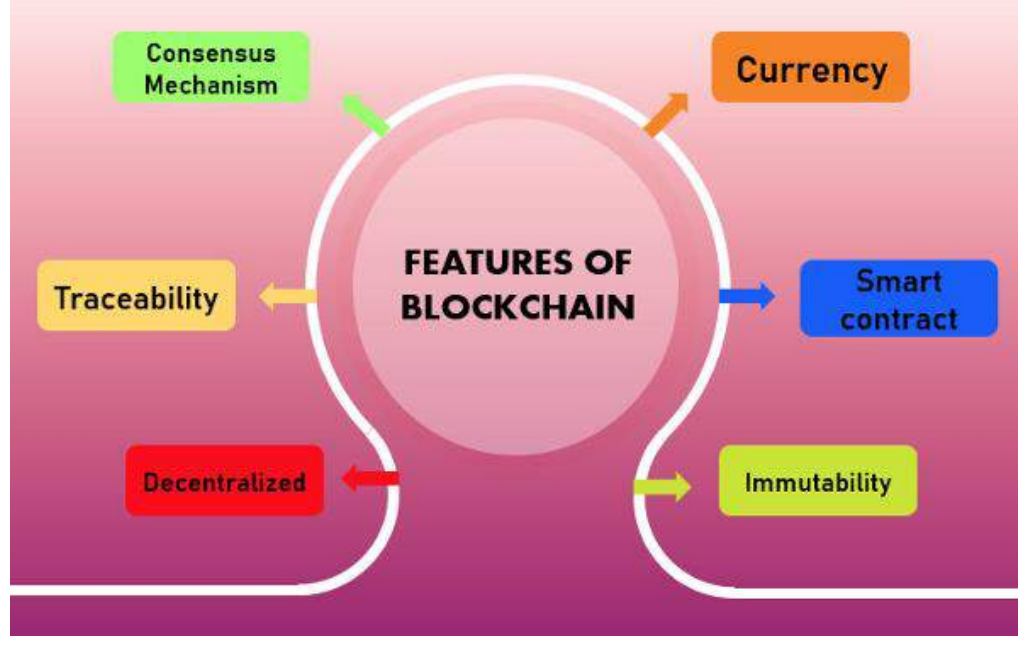

Figure 1 Blockchain Features

\section{Blockchain Applications in Education}

Blockchain is a technology that has applications in the world of learning at the institutional, group, individual, national, and even international levels. Where this applies to all types of terms of reference such as elementary schools to high schools, universities, colleges, fields of cooperation, scholarships, and knowledge. With trust migrating toward technology rather than institutions, technology is the main target compared to the old hierarchical structure. According to Donald Clark, this technology is very decentralized.

The following are some of the educational projects that are used throughout the blockchain-based world:

Sony Global Education (SGE): Is a platform that protects and also shares student records using a blockchain base. On this platform is a new educational platform raised by the IBM ministry. Sony's global education and Sony corporation announced that they will form a system on August 10, 217, which specifically blockchain technology will be applied in the education system. Sony will begin establishing its service offering in 2018, to bring 150000 from all over the world by being eager to help to begin the global mathematical challenges [26], [27], [28], [29].

Edgecoin: To ensure great regulation, edgecoin has a strategy in preparing daap, which aims to reduce efficiency, protect fraud, and also costs. This edgecoin speeds up the ends of the system and stores academic documents such as degrees. They don't need to pay official officials, so they can cut costs for students. Edgecoin is an innovative reform step in the total education trade because it can reduce disruption from non-current and unhealthy education, by validating its procedures and certifications. So they can survive all forms of damage, fraud, and loss in any form [21].

APPII: Is useful for ensuring the academic background and qualifications of teachers and students, by combining smart contracts and blockchain. This application is expected to maximize everyone's abilities. Users can create their profile and then an academic CV such as education history and transcripts [30].

Tutellus: A new blockchain platform, which hopes to solve the current cost of education for students in college, this platform will even pay students to study. USD 165 billion is a determined value, which was owned by online education in 2016. But it still cannot meet the needs of the education sector that exists throughout the world. This is not only about the number of unemployed graduates of $\mathbf{3 0 0}$ million people, but the increasing number of educated people but 
the fact that positions and job vacancies are gradually decreasing. The European part is expected to experience a balance between demand and supply of jobs by 80 percent, this will affect many sectors, especially the technology sector. This causes an increase in awareness of the field of education where educational institutions lack knowledge of something new that is in line with the company. Meanwhile, millions of people in developing countries are helped by their cell phones to be able to access the internet. They can access it because not everyone can access it. New methods are needed to help educational institutions solve the main problems on the market [31], [32], [33].

SuccessLife: Is the world's leading organizer of seminars, pioneering products, and workshops, coming from successful resources. SuccessLife is available for many people to use development both personally and professionally. SuccessLife is a blockchain company founded by Success Resources. Every month it will start a new business, which makes successlife its main target, around 543,000 businesses. Some residents work in small businesses, namely private businesses by more than 50 percent. Then there are 52 percent of small home-based businesses.

TeachMePlease (TMP): Providing a database of learning institutions for both online and offline schools, the programs available are very different from group learning classes for children ranging from individual sessions to private instructors. The program needs to work gently on data, such as assignments, solutions, courses, and updates. Compared to the TMP blockchain asking for fewer permissions, such as EOS or Ethereum will ask for public data disclosure. How does that make sense, like Hyperledger, also requires an open status that doesn't need to be questioned? "A quality platform, providing easy-to-use services for all our customers" [34], [35].

GradBase: A system based on bitcoin blockchain technology for verification of educational records. Gradbase provides a profile system that looks simple and inexpensive. This system has a QR code that will be entered into the student's CV. With Linkedln's support on expanding web browsers, it aims to improve the user experience. The Universe can enter the web application easily, to cancel qualifications, make updates in the appropriate procedures.

ODEM: Is a platform that allows students, teachers and related parties to connect in relevant services and courses. In addition, in obtaining a good education, ODEM has opportunities in the challenges faced by students. because using a framework supported by blockchain can improve education progress, low cost, and easy to handle [36].

Blockcerts: is a platform developed by MIT lab that collaborates with the education system for educational records. Blockcerts are used to verify official documents and publish them [37]. It also includes a wallet application that is used to encrypt student information. With the primary goal of producing valid and secure e-certificates using bitcoin blockchain technology [34], [35].

Parchment: is a forum used to assess academic qualifications, to process programs and produce certificates. Students can discuss academic performance with prospective employers easily [36]. This is the most widely accepted electronic credential service that allows students, academic institutions and employers to apply, verify and share their credentials easily and in a safe environment. Its framework has helped millions of people and thousands of colleges and universities transfer more than 30 million transcripts and other certificates worldwide [36].

Echolink: "Human Capital Link Skills Education Link" is an abbreviation of Echolink. Managing the relationship of educational entities such as teachers, students, and existing organizations is the goal of this blockchain-based system. This system stores data that has been verified from individuals, who are related to a professional career in a hash format, and the data cannot be changed on an unlicensed blockchain. All information contained in this system is provided by trusted sources. Further identified projects will be compared and related synthetics will be presented in this section [35], [37].

Origin-Stamp: is a safe time stamping, which has the aim to ensure security on digital data. This can be done by determining economic fingerprints for which data comes from clients, 
and ensuring that these fingerprints can be stored on the bitcoin blockchain technology permanently [29].

Further identified projects will be compared and related synthetics will be presented in the following sections:

\section{E. Comparative Secondary Studies}

Blockchain technology receives tremendous appreciation because of its progress and rapid miracle, it is no doubt because the appreciation is given by the whole world. However, we found only couples discussing the latest educational projects, we found it while looking for secondary studies.

First, we have recognized that in this study only a few recent projects can be explained briefly, the rest are not even touched and cannot be explained, this is because a report published by the European Commission was found. Second, a literature review that discusses the application of blockchain in the field of education, which was published in Springer.

\section{Research Methodology}

"The method of identifying, collecting, and interpreting all available research aimed at answering questions from certain studies" is the definition of a systematic literature review [38]. The guidelines provided by "Barbara Kitchenham" will be the guidelines we followed in conducting this research [39]. The results of this examination help us distinguish between the research gaps in mind and the research areas related to blockchain technology. SLR consists of 3 main stages according to Kitchenham, namely:

- Planning a review.

- Conduct a review.

- Report a review.

In completing this research, we will follow the steps above. Figure 2 shows the stages that will be divided into several more stages.

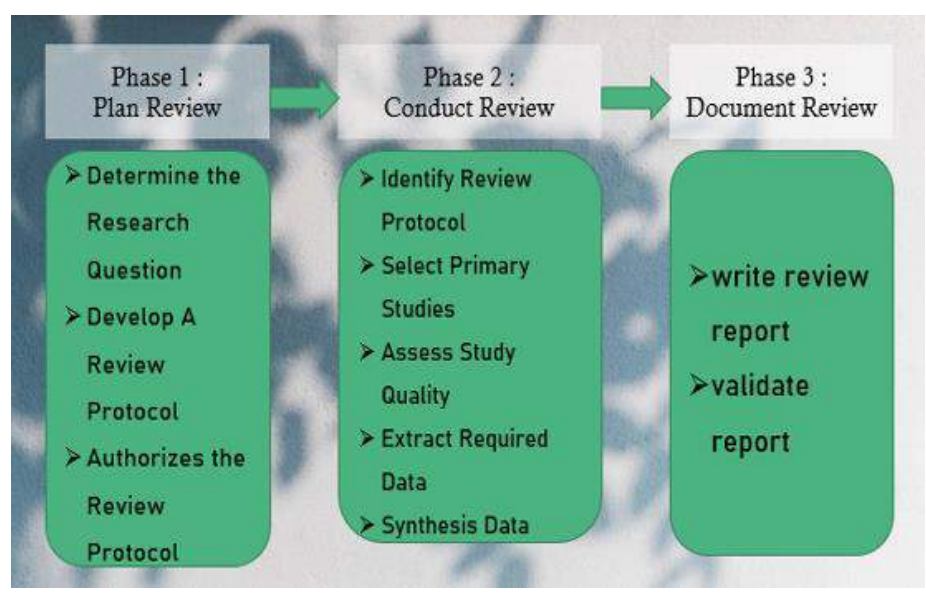

Figure 2 SLR Methodology
A. The Need to Perform SLR

ATM Vol 5, No. 1,January $2021: 46-61$ 
Based on the classification of this study, it was confirmed that some holes had not been discussed and had to be filled to obtain further advice, it was discovered after analyzing this study. During the process of conducting this research, only a few studies were found that discussed education projects based on blockchain. But it was not done in full voice in a single time paper. Furthermore, this leads us to conduct quantitative and appropriate research so that we can achieve our goals.

\section{B. Research questions}

Raising research questions is the first part of the review literature review:

RQ1: What are the educational projects based on blockchain technology? (Objective: To describe several blockchain technology projects that can be developed for education)

RQ2: In which projects are the protocols used?

RQ3: In these projects what blockchain features will be used? (aiming to describe the blockchain features used in the project and expand the blockchain features not discussed in other studies.)

RQ4: By using the blockchain feature, what services are offered by existing educational projects? (aim: To observe the same or different services to improve the application of blockchain technology in education)

\section{Search Strategy}

In the education sector blockchain plays an important role. Through google scholar, we study different analysis papers. We tend to search for keywords such as "Blockchain", "review", and "education" on Google Scholar. 200 subjects were found on this subject. After studying the paper, we selected several papers that were related to the keywords we used. Then examine various research papers, after which report an article that has been published in various conferences and journals.

\section{Inclusion and Exclusion Criteria}

35 papers that have been collected, relating to keywords and research questions to be included. We selected 11 main studies after reading abstracts and conclusions to separate the data, the study of a blockchain-based education project that was suitable for the needs of the latest educational trends. Another paper was omitted. After all, it had no connection with the topic "Project-based on technology education" because it discussed "Blockchain Technology in Education".

TABEL I. PAPER WITH REFERENCE ID

\begin{tabular}{|c|c|c|}
\hline ID & Publish Year & Paper name \\
\hline [39] & 2017 & Stampery Blockchain Timestamping Architecture (BTA)-Version 6 \\
\hline [36] & 2018 & $\begin{array}{c}\text { Exploring blockchain technology and its potential applications for education } \\
\text { Blockchain Based Professional Networking and Recruiting Platform }\end{array}$ \\
\hline [37] & 2018 & $\begin{array}{c}\text { CredenceLedger: A Permissioned blockchain for Verifiable Academic } \\
\text { Credentials }\end{array}$ \\
\hline$[34]$ & 2018 & Blockchain for education: lifelong learning passport \\
\hline [40] & 2018 & Disciplina: blockchain for Education \\
\hline$[33]$ & 2018 & Pemanfaatan Teknologi Blockchain pada Program Sertifikasi Dosen \\
\hline$[10]$ & 2019 & \\
\hline
\end{tabular}




\begin{tabular}{|c|c|c|}
\hline [41] & 2019 & $\begin{array}{c}\text { Design Framework on Tertiary Education System in Indonesia Using } \\
\text { Blockchain Technology }\end{array}$ \\
\hline [9] & 2020 & Blockchain Technology into Gamification on Education \\
\hline$[16]$ & 2020 & Application of Blockchain Technology for iLearning Student Assessment. \\
\hline [11] & 2020 & $\begin{array}{c}\text { The Role Of Blockchain As A Security Support For Student Profiles In } \\
\text { Technology Education System }\end{array}$ \\
\hline
\end{tabular}

\section{E. Data Extraction}

The selected study data will then enter the extraction stage and then begin to be applied. At this stage, appropriate data will be selected from the main study because it focuses on the blockchain-based education project and its basic features have been followed by the identified project. For analytical purposes, the extracted data will be used. There are 12 main studies covering educational projects [40], [21], [38], [35], [41], [34], [10], [42], [9], [17], [11].

\section{F. Publication Trends}

The adoption and progress of blockchain technology have increased at a higher level, this has happened since 2008 where blockchain research was conducted. However, it was in 2017 that the first paper published on the blockchain-based education project was discovered. But no papers were found, which discussed explicitly and centered on the educational projects that had been identified

\section{Result}

In the results section, 4 sections have been divided. The first part is a grouping or classifying several blockchain projects related to education. The second part explains the features used for these projects. The third part focuses on the general and unusual services of the education project that have been identified, and the fourth section explains the future directions.

A. RQ1: What are the educational projects based on blockchain technology?

Technology has produced several methods and new educational models, this is due to a very rapid revolution in technology [43]. In the world of education, blockchain technology has brought change (Revolution). Our first research question relates to the project selected by studying existing review papers. The following is shown in figure 3. 


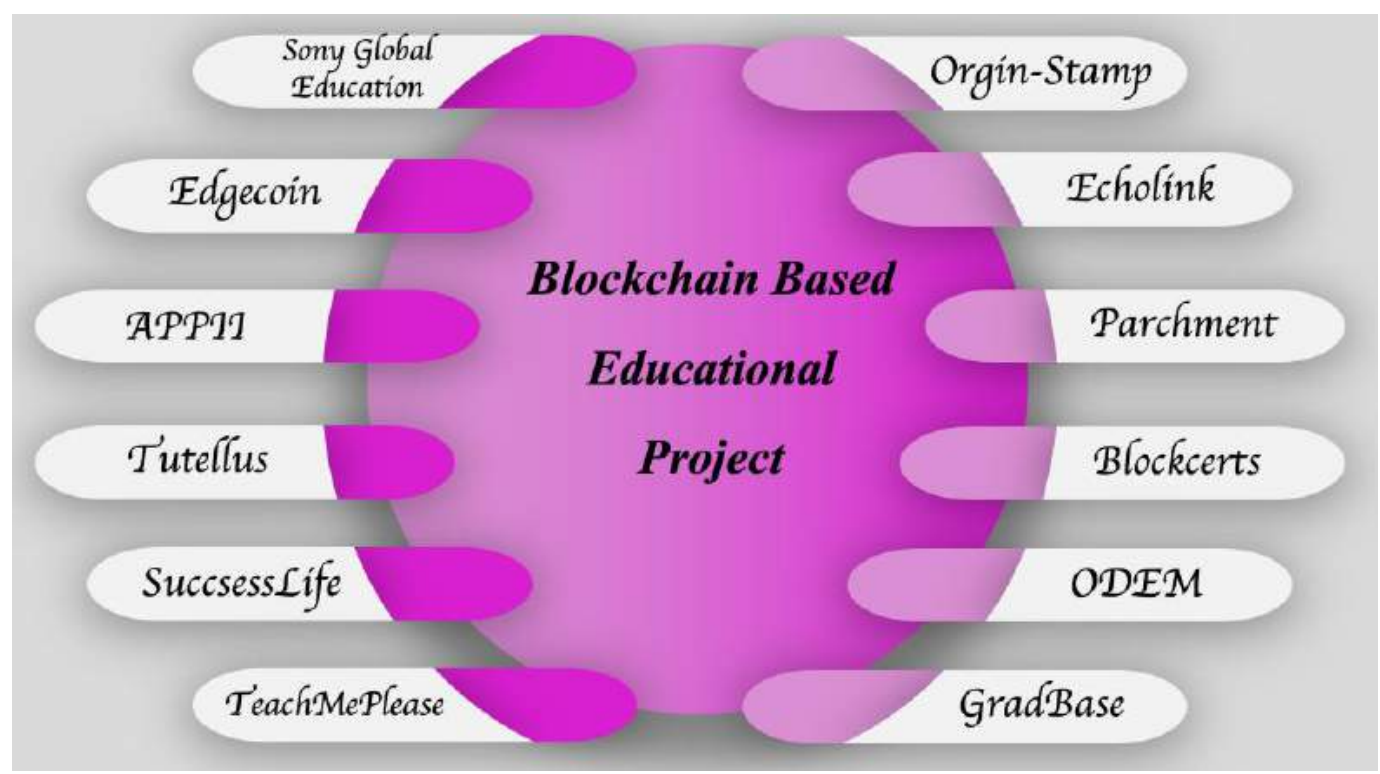

Fig 3. Blockchain-based Education Project

These are Sony Global Education Project, Edge-coin, APPII, Tutellus, Success Life, Teach Me Please, GradeBase, ODEM, Blockcerts, Echolink, Parchment and Origin-Stamp.

Through the general introduction, it makes us interested in finding out the reasons behind this evolution. Our contributions are:

- Why do we need blockchain based-education projects?

The reason is that individuals, universities, and entrepreneurs are currently facing a lot of challenges relating to verification of education, fraud, comparing the same degree in different educational institutions and translating problems [44]. Also, as for authenticating, printing, storing certification and degrees are high costs for schools and universities in various parts of the world meanwhile e-learning is becoming increasingly popular, validation of skills and verification of certifications remain because all institutions, companies, and countries do not have certain guidelines to be accepted by the company. Large gaps in the workforce occur due to the inability to validate skills. Every individual need's something that can make it easier for them to apply for a job and companies that can hire people who have met the requirements, that can happen if the multibillion-dollar education industry finds a solution to make it easier for them to verify, validate and aggregate individual "learning notes".

Therefore we need a solution to solve every challenge that exists. So, this blockchainbased project becomes a solution to complete the challenge, because the blockchain is very fast, safe, and also cost-efficient in compiling data and records such as e-learning certificates, college degrees, and others.

B. RQ2: In which projects are the protocols used?

By following blockchain technology, all educational projects that have been built have the main goal of which is to examine all problems and obstacles that come as a barrier and promote a real sense of education, this can provide a rational solution and be a solution to all existing problems. The main problems that arise regarding the issue of education are too many traditional documents, lack of security or protection of educational data, how to store data that is still inadequate, the process of certification is inefficient and takes a long time, as well as storing old records and not can be changed, in others [45]. Therefore, the existence of blockchain technology in education will provide convenience in conducting verification and validation, which initially takes a very long and tedious process to be fast to save valuable time and money [42]. Poverty is a root and a common cause of illiteracy in third world countries. The most significant achievements of the model are that they provide 
opportunities to improve or enhance economic positions and provide knowledge skills to students [46].

TABEL II. EDUCATION AND TECHNOLOGY PROJECT USED

\begin{tabular}{|c|c|c|}
\hline No & Model's & Technology \\
\hline 1 & Edge-coin & Proof of Stake (PoS) \\
\hline 2 & Sorry Global Education Project & Hyperledger \\
\hline 3 & Tutellus & Sharding \\
\hline 4 & TeachMePlease & DOLS \\
\hline 5 & Success Life & POW \\
\hline 6 & Blockcerts & POW \\
\hline 7 & GradeBase & PoS \\
\hline 8 & Echolink & \\
\hline
\end{tabular}

Edge-Coin stores educational documents in its blocks such as course and degree certifications, then the third party will immediately confirm their validity. This can reduce costs incurred for students because they no longer need to pay for notaries. It can also reduce costs incurred for the institution because it eliminates the documents discussed in section II and bureaucracy.

POS: Echolink and Edge-Coin have used something contrary to Proof of Work, Proof of Stake (PoS). PoS is one of the nodes selected for most nodes that have not been selected by the network at their age and quantity. To monitor the site selected certain individuals, so that Edgecoin and Echolink can handle the platform faster, smart contract transmission goes faster [47].

POW: blockchain-based education projects that use Proof of Work (POW) are gradbase, origin-stamp, and blockcerts. The purpose of this protocol is to validate transactions. Using this protocol, miners compete with each other, to complete transactions by solving cryptographic puzzles and getting and collecting prizes received afterward which require more time during processing.

DPoS: A blockchain-based education project that uses the DPoS protocol is TeachMePlease (TMP). Delegated Proof of Stake (DPoS) has an understanding of the consensus mechanism algorithm which maintains an indisputable agreement regarding authorizing transactions, the reality across the network, and providing a digital democratic platform. Because of its performance and exceptional reputation lisk has chosen it as the chosen protocol. Delegated evidence of ownership that is by using real-time voting, to reach consensus will be combined with the naming scheme. This can be seen from the comparison between the consensus protocol which has the smallest number compared to all existing consensus because it is the most cooperative of all that exists. What happens to the network there is influenced by each token holder.

The private and public layers are part of the teachmeplease blockchain architecture. Private Layer: Used to store material protected by copyright and personal data [34]. Public Layer: Used to provide access to data that verifies network data storage constraints and personal data integrity [34].

BOLT: To make personal and professional developments available to many people, which are a source of success in building and succeeding the blockchain company, successlife. successlife uses the BOLT technology protocol. This technology can enable provenance and create entries on the blockchain.

- The blockchain level has captured the user's search history and interest in the level. This process can provide a better understanding and knowledge about student interests.

- The trainer can identify the number of times students are asked for a demonstration assisted by making entries at the blockchain learning level that can be seen from the demo step. 
Hyperledger: a multipurpose business platform that supports various educational institutions in storing digital certificates and education records is sony global helpledger. Providing Application Programming Interface $(\mathrm{API})$ to be a solution that aims to control user rights for educational institutions and adjust student data collection and record attributes [6], [28]

Separating the standard PoW transaction process that is being carried out into three phases can solve the problem using Sony hyperledger. The three phases are: Order blocks, build blocks, and validate each of the existing blocks.

Sharding: is a type of database partitioning that separates databases from larger ones into smaller, more manageable, and faster parts called data fractions [22]. It often happens, shard data is relatively easier. Many transactions will be validated simultaneously using this method on the ethereum network. This sharding allows companies to delegate their different tasks, due to the presence of tutellus, so that they can maximize the capacity for their educational functions [48].

A total of more than 100 users have proven that tutellus has become very popular in the Spanish-speaking world. Tutellus also provides educational video courses for more than 150 videos. There is a market for applications that can reduce loan costs, which is no doubt because there is a market, with many students taking loans ranging from 20,000 to 60,000 dollars.

\section{RQ3: In these projects, what blockchain features will be used?}

To describe the blockchain features used in these projects, is the aim of this research question. In these projects, the blockchain features that are commonly used are listed in table III.

TABEL III. FEATURES USED BY THIS PROJECT

\begin{tabular}{|c|c|c|c|c|c|c|c|c|c|c|c|c|c|}
\hline $\begin{array}{c}\text { Projects / } \\
\text { Feature }\end{array}$ & SGE & $\begin{array}{c}\text { Edge } \\
\text { coin }\end{array}$ & APPII & $\begin{array}{c}\text { Success } \\
\text { Life }\end{array}$ & TMP & Tutellus & $\begin{array}{c}\text { Grade } \\
\text { Base }\end{array}$ & ODEM & Blockcerts & $\begin{array}{c}\text { Parch } \\
\text { ment }\end{array}$ & $\begin{array}{c}\text { Echo } \\
\text { Link }\end{array}$ & $\begin{array}{l}\text { Origin } \\
\text { Stamp }\end{array}$ \\
\hline Decentralize & $\sqrt{ }$ & $\sqrt{ }$ & $\sqrt{ }$ & $\sqrt{ }$ & $\sqrt{ }$ & $\sqrt{ }$ & $\sqrt{ }$ & $\sqrt{ }$ & $\sqrt{ }$ & $\sqrt{ }$ & $\sqrt{ }$ & $\sqrt{ }$ \\
\hline Traceability & $\sqrt{ }$ & $\sqrt{ }$ & & $\sqrt{ }$ & $\sqrt{ }$ & $\sqrt{ }$ & $\sqrt{ }$ & & $\sqrt{ }$ & & $\sqrt{ }$ & $\sqrt{ }$ \\
\hline Currency & & $\sqrt{ }$ & $\sqrt{ }$ & $\sqrt{ }$ & & $\sqrt{ }$ & & $\sqrt{ }$ & $\sqrt{ }$ & $\sqrt{ }$ & & \\
\hline Immutability & & $\sqrt{ }$ & $\sqrt{ }$ & $\sqrt{ }$ & $\sqrt{ }$ & $\sqrt{ }$ & $\sqrt{ }$ & $\sqrt{ }$ & $\sqrt{ }$ & $\sqrt{ }$ & $\sqrt{ }$ & $\sqrt{ }$ \\
\hline $\begin{array}{c}\text { Censorship } \\
\text { Resistant }\end{array}$ & & $\sqrt{ }$ & & $\sqrt{ }$ & $\sqrt{ }$ & $\sqrt{ }$ & $\sqrt{ }$ & & $\sqrt{ }$ & & $\sqrt{ }$ & $\sqrt{ }$ \\
\hline $\begin{array}{c}\text { Transaction } \\
\text { Rate }\end{array}$ & $\sqrt{ }$ & $\sqrt{ }$ & & $\sqrt{ }$ & $\sqrt{ }$ & $\sqrt{ }$ & $\sqrt{ }$ & & $\sqrt{ }$ & & $\sqrt{ }$ & $\sqrt{ }$ \\
\hline Anonymity & $\sqrt{ }$ & & & $\sqrt{ }$ & & & & & & & & \\
\hline Open Source & $\sqrt{ }$ & $\sqrt{ }$ & & $\sqrt{ }$ & & $\sqrt{ }$ & & & $\sqrt{ }$ & & & \\
\hline
\end{tabular}

- Unlike stores with centralized power, these blockchain-based educational projects are fully decentralized. By storing all of this educational project data in a decentralized blockchain network will provide independence and transparency in the system. The reason is because the blockchain is an open-source of information, therefore data sets must be stored accurately and honestly. Also, a decentralized management system will provide technical facilities to certify certification individually and to employers or institutions to certify quality just by clicking, rather than using methods that will take time. Blockchain technology will validate new blocks into the distributed ledgers, so this technology uses a consensus mechanism. Projects that have been recognized use a consensus mechanism namely: POS, POW and DPOS. 
- This educational project focuses on the enduring record of the intention, the record that cannot be changed. If information has been stored on the network it cannot be changed, unless 51 percent of the total nodes agree to make changes.

- This blockchain-based education project is open source. These projects are always open to everyone, the environment is supportive and can ensure that transparency is complete.

- Anonymous data is only provided by SGE. This feature can reverse traceability. Without using any encryption method users can store data that is private.

- Hold censorship means no rewriting and no changes to the data. But everyone can still see every transaction. Because the anonymity of SGE (Sony Global Education) features can censor transactions.

D. RQ4: By using the blockchain feature, what services are offered by existing educational projects?

To identify services offered by educational projects that use the blockchain feature are the main objectives of this research question. The first thing we did was to study seriously about the research project that was given. We find out about the results given in Figure 3 below after conducting the in-depth analysis.

We will present a comparison table between blockchain-based education projects in table IV. Of the many benefits derived from all technologies, some can be combined and that can produce even better results.

TABEL IV. SERVICES PROVIDE BY PROJECT

\begin{tabular}{|l|l|l|l|l|l|l|l|l|l|l|l|l|}
\hline Projects / Service & SGE & $\begin{array}{l}\text { Edge } \\
\text { coin }\end{array}$ & APPII & $\begin{array}{l}\text { Success } \\
\text { Life }\end{array}$ & TMP & Tutellus & $\begin{array}{l}\text { Grade } \\
\text { Base }\end{array}$ & ODEM & Blockcerts & $\begin{array}{l}\text { Parch } \\
\text { ment }\end{array}$ & $\begin{array}{l}\text { Echo } \\
\text { Link }\end{array}$ & $\begin{array}{l}\text { Origin } \\
\text { Stamp }\end{array}$ \\
\hline Content Library & $\sqrt{ }$ & $\sqrt{ }$ & & $\sqrt{ }$ & $\sqrt{ }$ & $\sqrt{ }$ & & & & & & \\
\hline Store personal data & $\sqrt{ }$ & $\sqrt{ }$ & $\sqrt{ }$ & $\sqrt{ }$ & $\sqrt{ }$ & & $\sqrt{ }$ & & $\sqrt{ }$ & & $\sqrt{ }$ & $\sqrt{ }$ \\
\hline E-Certficate & $\sqrt{ }$ & $\sqrt{ }$ & $\sqrt{ }$ & $\sqrt{ }$ & $\sqrt{ }$ & & $\sqrt{ }$ & & $\sqrt{ }$ & $\sqrt{ }$ & & \\
\hline Scoring system & $\sqrt{ }$ & & & & & & & & & & & \\
\hline B2B approach & $\sqrt{ }$ & $\sqrt{ }$ & $\sqrt{ }$ & $\sqrt{ }$ & & $\sqrt{ }$ & & $\sqrt{ }$ & & & $\sqrt{ }$ & \\
\hline Token system & & $\sqrt{ }$ & & $\sqrt{ }$ & & $\sqrt{ }$ & $\sqrt{ }$ & $\sqrt{ }$ & $\sqrt{ }$ & & & \\
\hline Cooperative learning & & $\sqrt{ }$ & $\sqrt{ }$ & & & $\sqrt{ }$ & & & & $\sqrt{ }$ & & \\
\hline Job opportunities & $\sqrt{ }$ & & $\sqrt{ }$ & & & & $\sqrt{ }$ & & & & & \\
\hline $\begin{array}{l}\text { Providing Feedback } \\
\text { services }\end{array}$ & & & & & $\sqrt{ }$ & & & & & & \\
\hline
\end{tabular}

There are so many benefits in educational projects, namely: First of all, a decentralized approach that can solve many problems regarding, document reduction, employment, and security problems. To process the several billion transactions that occur between devices, it is fundamentally adopted as a standard blockchain that is useful for reducing costs that have to do with maintaining large, centralized data centers and adjusting to capacity and calculation requirements that exceed billions of devices. This will prevent and avoid a single network code from collapsing all systems. Another aim of this project is that educational projects are used to continue to support the small-scale loT system and connect students and teachers in the past few years. However, it has not been able to provide needs that are used to expand the blockchain system in education in the future.

TABLE V. SERVICE OFFERED BY BLOCKCHAIN FEATURES

Services offered by Projects

Blockchain Features

ATM Vol 5, No. 1,January 2021 : 46-61 


\begin{tabular}{|c|c|}
\hline Content Library & Decentralized and Transaction Rate \\
\hline Store personal data & Anonymity \\
\hline E-Certificate & Transaction Rate \\
\hline Scoring system & Smart Contract \\
\hline B2B approach & Smart Contract \\
\hline Token system & Consensus Mechanism, Smart Contract and Currency \\
\hline Cooperative learning & Decentralized and Smart Contract \\
\hline Job opportunities & Smart Contract \\
\hline Providing Feedback services & Traceability \\
\hline
\end{tabular}

Content Library this is a great note that offers blockchain-based educational projects using the blockchain features of the decentralized blockchain and transaction level features. In this service if the customer leaves content at one point on a device, then on another device he will continue to study it, this is because this service is platform independent.

Storing Personal Data This means that customers can save their data in 2 ways, namely the encryption and anonymous methods. The encryption method is that all transactions that are stored on the network show everyone but in the form of encryption. Data is encrypted using different codes, and all projects will provide the same data security like this. Anonymous method, this technique is only used for SGE projects. Unknown transactions are kept in a tightly closed meeting, no one can see or know about these transactions other than the customer himself.

E-Certificate is an official certificate using the transaction rate of the blockchain feature we will receive because it is provided by the existing blockchain. An e-certificate is a digital certificate that takes a digital signature, by means of client can achieve it using private keys and public keys.

Rating System by using the blockchain feature, smart contact, grades will be given according to the achievements obtained by students. Only teachmeplease and sony global provide assessment system services. If during the process of evaluating the achievement produced by students meet the requirements then declared extraordinary.

B2B approach blockchain helps this system because of its decentralized nature, this system is a direct contact that starts with one business then continues to the next business. These two businesses are connected. By utilizing the blockchain feature, namely smart contact, this system service is offered.

Token System with the ultimate goal of the transaction and as a reward, this token framework can be used. A variety of components and methods are used for this, the service is offered by three features namely the consensus mechanism feature, smart contract features and currency features.

Cooperative Learning means that people learn together in the same place. They create new thinking and talk about things about block-chain-based education projects, which can use decentralized blockchain features and smart contract features.

Job Opportunities This block-chain based education project can provide employment opportunities to someone. They provide this job opportunity to people who are skilled and also certified. That way, using smart blockchain contracts can reduce unemployment.

Provide Feedback service the blockchain-based education project, tutellus, provides facilities for people to provide feedback, by using the search feature.

\section{Conclusion}


In conducting this research, using a systematic literature approach in mapping out can be useful. In this case, it is known which projects are suitable for blockchain-based education and a comparison of the features of this project has been presented. Common problems that occur in the world of education can be resolved because of the implementation of blockchain technology.

These education projects have the benefit of a decentralized approach. Where this can solve a lot of problems regarding document reduction, job problems, and security. This can prevent and prevent a single network code from crashing all systems.

This technology goes through an evaluation process that is at an early experimental stage. This technology can bring even more innovations in the future. Therefore, more extensive development is still needed so that this technology is more advanced and has other benefits for the world of education.

\section{Acknowledgement}

The author would like to thank the University of Raharja and all those who have allowed the author to do this writing and have also given advice, support, and guidance so that this writing can be completed properly.

\section{Reference}

[1] S. Nakamoto, "Bitcoin: A peer-to-peer electronic cash system," http://bitcoin. org/bitcoin. pdf," 2008.

[2] M. Conoscenti, A. Vetro, and J. C. De Martin, "Blockchain for the Internet of Things: A systematic literature review," in 2016 IEEE/ACS 13th International Conference of Computer Systems and Applications (AICCSA), 2016, pp. 1-6.

[3] M. Crosby, P. Pattanayak, S. Verma, and V. Kalyanaraman, "Blockchain technology: Beyond bitcoin," Appl. Innov., vol. 2, no. 6-10, p. 71, 2016.

[4] D. Bhowmik and T. Feng, "The multimedia blockchain: A distributed and tamper-proof media transaction framework," in 2017 22nd International Conference on Digital Signal Processing (DSP), 2017, pp. 1-5.

[5] H. Yumna, M. M. Khan, M. Ikram, and S. Ilyas, "Use of Blockchain in Education: A Systematic Literature Review," in Asian Conference on Intelligent Information and Database Systems, 2019, pp. 191-202.

[6] M. B. Hoy, "An introduction to the blockchain and its implications for libraries and medicine," Med. Ref. Serv. Q., vol. 36, no. 3, pp. 273-279, 2017.

[7] J. Yli-Huumo, D. Ko, S. Choi, S. Park, and K. Smolander, "Where is current research on blockchain technology?-a systematic review," PLoS One, vol. 11, no. 10, p. e0163477, 2016.

[8] F. Agustin, Q. Aini, A. Khoirunisa, and E. A. Nabila, "Utilization of Blockchain Technology for Management E-Certificate Open Journal System," Aptisi Trans. Manag., vol. 4, no. 2, pp. 134-139, 2020.

[9] Q. Aini, U. Rahardja, and A. Khoirunisa, "Blockchain Technology into Gamification on Education," IJCCS (Indonesian J. Comput. Cybern. Syst., vol. 14, no. 2.

[10] M. Yusup, Q. Aini, D. Apriani, and P. Nursaputri, "PEMANFAATAN TEKNOLOGI BLOCKCHAIN PADA PROGRAM SERTIFIKASI DOSEN," in SENSITIf: Seminar Nasional Sistem Informasi dan Teknologi Informasi, 2019, pp. 365-371.

[11] P. A. Sunarya, U. Rahardja, L. Sunarya, and M. Hardini, "The Role Of Blockchain As A Security Support For Student Profiles In Technology Education Systems," InfoTekJar J. Nas. Inform. dan Teknol. Jar., vol. 4, no. 2, pp. 13-17, 2020.

[12] S. Makridakis, A. Polemitis, G. Giaglis, and S. Louca, "Blockchain: the next breakthrough in the rapid progress of Al," Artif. Intell. Trends Appl., pp. 197-219, 2018.

[13] G. Albeanu, "Blockchain technology and education," in The 12th International Conference on Virtual Learning ICVL, 2017, pp. 271-275.

[14] J. Rooksby and K. Dimitrov, "Trustless education? A blockchain system for university 
grades," in New Value Transactions: Understanding and Designing for Distributed Autonomous Organisations, Workshop at DIS, 2017.

[15] F. Agustin, S. Syafnidawati, N. P. Lestari Santoso, and O. G. Amrikhasanah, "Blockchainbased Decentralized Distribution Management in E-Journals," Aptisi Trans. Manag., vol. 4, no. 2, pp. 107-113, 2020.

[16] M. Vukolić, "The quest for scalable blockchain fabric: Proof-of-work vs. BFT replication," in International workshop on open problems in network security, 2015, pp. 112-125.

[17] S. Sudaryono, Q. Aini, N. Lutfiani, F. Hanafi, and U. Rahardja, "Application of Blockchain Technology for iLearning Student Assessment," IJCCS (Indonesian J. Comput. Cybern. Syst., vol. 14, no. 2.

[18] K. Biswas and V. Muthukkumarasamy, "Securing smart cities using blockchain technology," in 2016 IEEE 18th international conference on high performance computing and communications; IEEE 14th international conference on smart city; IEEE 2nd international conference on data science and systems (HPCC/SmartCity/DSS), 2016, pp. 1392-1393.

[19] M. Alharby and A. van Moorsel, "Blocksim: a simulation framework for blockchain systems," ACM SIGMETRICS Perform. Eval. Rev., vol. 46, no. 3, pp. 135-138, 2019.

[20] A. Adiyanto and R. Febrianto, "Authentication Of Transaction Process In E-marketplace Based On Blockchain technology," Aptisi Trans. Technopreneursh., vol. 2, no. 1, pp. 6874, 2020.

[21] G. Chen, B. Xu, M. Lu, and N.-S. Chen, "Exploring blockchain technology and its potential applications for education,” Smart Learn. Environ., vol. 5, no. 1, p. 1, 2018.

[22] I.-C. Lin and T.-C. Liao, "A survey of blockchain security issues and challenges.," IJ Netw. Secur., vol. 19, no. 5, pp. 653-659, 2017.

[23] A. Grech and A. F. Camilleri, "Blockchain in education." Luxembourg: Publications Office of the European Union, 2017.

[24] E. P. Harahap, Q. Aini, and R. K. Anam, "PEMANFAATAN TEKNOLOGI BLOCKCHAIN PADA PLATFORM CROWDFUNDING," Technomedia J., vol. 4, no. 2, pp. 199-210, 2020.

[25] D. Mohanty, "Ethereum Use Cases," in Ethereum for Architects and Developers, Springer, 2018, pp. 203-243.

[26] M. Turkanović, M. Hölbl, K. Košič, M. Heričko, and A. Kamišalić, "EduCTX: A blockchainbased higher education credit platform," IEEE access, vol. 6, pp. 5112-5127, 2018.

[27] M. Sharples and J. Domingue, "The blockchain and kudos: A distributed system for educational record, reputation and reward," in European conference on technology enhanced learning, 2016, pp. 490-496.

[28] P. Ocheja, B. Flanagan, and H. Ogata, "Connecting decentralized learning records: a blockchain based learning analytics platform," in Proceedings of the 8th international conference on learning analytics and knowledge, 2018, pp. 265-269.

[29] T. Hepp, A. Schoenhals, C. Gondek, and B. Gipp, "OriginStamp: A blockchain-backed system for decentralized trusted timestamping," it-Information Technol., vol. 60, no. 5-6, pp. 273-281, 2018.

[30] "9 Blockchain Education Companies to Know | Built In." https://builtin.com/blockchain/blockchain-education (accessed Aug. 23, 2020).

[31] C. Soucy, "The Changing Tide of Corporate Social Responsibility," 2018.

[32] E. Martos Carrion, "Historico-Sociological Analysis of the Sharing Economy from its Early Digital Foundations to the Present," 2018.

[33] J. Martínez-Polo, J. T. Martínez-Sánchez, and J. M. N. Vivó, "Participation and sharing economy: The spanish case of\# compartirmola," in Entrepreneurship, Business and Economics-Vol. 1, Springer, 2016, pp. 15-22.

[34] K. Kuvshinov, J. Mostovoy, and I. Nikiforov, "Disciplina: Blockchain for Education.” 2018.

[35] R. Arenas and P. Fernandez, "CredenceLedger: a permissioned blockchain for verifiable academic credentials," in 2018 IEEE International Conference on Engineering, Technology and Innovation (ICE/ITMC), 2018, pp. 1-6.

[36] T. Alam and M. Benaida, "Blockchain and Internet of Things in Higher Education," Univers. J. Educ. Res., vol. 8, no. 5, pp. 2164-2174, 2020.

[37] A. Williams and E. Dolan, "Application of Blockchain Technology in e-LoA Technopreneurship Journal," Aptisi Trans. Technopreneursh., vol. 2, no. 1, pp. 98-103, 2020. 
[38] S. Chan, "Blockchain Based Professional Networking and Recruiting Platform." .

[39] B. Kitchenham and S. Charters, "Guidelines for performing systematic literature reviews in software engineering," 2007.

[40] A. S. de P. Crespo and L. I. C. García, "Stampery Blockchain Timestamping Architecture (BTA)-Version 6," arXiv Prepr. arXiv1711.04709, 2017.

[41] W. Gräther, S. Kolvenbach, R. Ruland, J. Schütte, C. Torres, and F. Wendland, "Blockchain for education: lifelong learning passport," in Proceedings of 1st ERCIM Blockchain Workshop 2018, 2018.

[42] U. Rahardja, A. N. Hidayanto, T. Hariguna, and Q. Aini, "Design Framework on Tertiary Education System in Indonesia Using Blockchain Technology," in 2019 7th International Conference on Cyber and IT Service Management (CITSM), 2019, vol. 7, pp. 1-4.

[43] U. Rahardja, Q. Aini, Y. I. Graha, and M. R. Tangkaw, "Gamification Framework Design of Management Education and Development in Industrial Revolution 4.0," in Journal of Physics: Conference Series, 2019, vol. 1364, no. 1, p. 12035.

[44] H. Elendner, S. Trimborn, B. Ong, and T. M. Lee, "The cross-section of crypto-currencies as financial assets: An overview," SFB 649 Discussion paper, 2016.

[45] D. J. Skiba, "The potential of Blockchain in education and health care," Nurs. Educ. Perspect., vol. 38, no. 4, pp. 220-221, 2017.

[46] Z. Zheng, S. Xie, H. Dai, X. Chen, and H. Wang, "An overview of blockchain technology: Architecture, consensus, and future trends," in 2017 IEEE international congress on big data (BigData congress), 2017, pp. 557-564.

[47] A. Kiayias, A. Russell, B. David, and R. Oliynykov, "Ouroboros: A provably secure proofof-stake blockchain protocol," in Annual International Cryptology Conference, 2017, pp. 357-388.

[48] L. Luu, V. Narayanan, C. Zheng, K. Baweja, S. Gilbert, and P. Saxena, "A secure sharding protocol for open blockchains," in Proceedings of the 2016 ACM SIGSAC Conference on Computer and Communications Security, 2016, pp. 17-30. 